\title{
INVESTIGACIONES
}

\section{La relación con los demás y la motivación en un Aprendizaje Basado en Proyectos}

\author{
Relatedness and motivation in a Project-based learning
}

\author{
Ana María Botella Nicolás ${ }^{a}$, Pablo Ramos Ramos ${ }^{b}$ \\ ${ }^{a}$ Departamento de Didáctica de la Expresión Musical, Plástica y Corporal. \\ Universitat de València, España. \\ ana.maria.botella@uv.es \\ ${ }^{b}$ IES Luis Vives de Valencia, España. \\ pablo.ramos.ramos@gmail.com
}

\section{RESUMEN}

La relación entre pares es esencial en el desarrollo de metodologías activas como el Aprendizaje Basado en Proyectos (ABP). Su importancia no solo reside en la creación de un contexto eficiente de trabajo, sino en el fomento de la motivación del alumno. Para entender cómo funciona esta última, la teoría de la autodeterminación ofrece un modelo en el que el desarrollo de la motivación intrínseca se asocia al fomento de tres necesidades psicológicas básicas: autonomía, competencia y relación con los demás.

El objetivo de este trabajo es analizar cómo funcionó la relación con los demás durante un ABP de naturaleza artística en dos grupos de educación secundaria. Los resultados muestran que la relación con los compañeros fue uno de los elementos más motivadores del mismo y que esta necesidad psicológica se vio favorecida por el desarrollo de las otras dos, autonomía y competencia.
\end{abstract}

Palabras claves: aprendizaje activo, aprendizaje en grupo, autonomía, educación secundaria.

\begin{abstract}
Peer relationship is an essential element for the development of an active learning such as Project-based learning (PBL). The importance of that element goes beyond the creation of an efficient work environment, it is also important to foster motivation. Self-determination theory suggests that the development of intrinsic motivation is associated with three basic psychological needs: autonomy, competence and relatedness.

The objective of the present study is to analyze how relatedness was developed during a PBL in two groups of secondary education with different autonomy degrees. An action research was undertaken in order to foster the three psychological needs during the project. The students were interviewed about the elements that supported their motivation. Results of the content analysis found that peer relationship was one of the most motivational element of the project and that it was promoted by the other psychological needs, autonomy and competence.
\end{abstract}

Key words: activity learning, group learning, autonomy, secondary education. 


\section{INTRODUCCIÓN}

El Aprendizaje Basado en Proyectos (ABP) es una metodología activa en la que el currículo de una o varias asignaturas se desarrolla a través de un trabajo de larga duración que parte de una pregunta inicial (Thomas, 2000). Dado que todos los participantes deben contribuir a la realización de un resultado común, denominado producto, el ABP es una forma colaborativa de aprendizaje (Helle, Tynjälä y Olkinuora, 2006) que permite un aprendizaje en profundidad (Mergendoller, Markham, Ravitz y Larmer, 2006).

La importancia de la relación entre pares en este tipo de metodología radica en que, en situaciones de cooperación, los individuos perciben que pueden alcanzar sus metas solo si el resto de los miembros del grupo también lo hace (Qin, Johnson y Johnson, 1995). En este sentido, varios autores (Stefanou, Stolk, Prince, Chen y Lord, 2013; Kokotsaki, Menzies y Wiggins, 2016) señalan que una de las características más importantes del ABP es precisamente la colaboración entre alumnos (alumnos y profesores, y alumnos con otros miembros de la comunidad).

Para Ryan, Stiller y Lynch (1994), los seres humanos de cualquier edad son más felices y más capaces de desarrollar sus talentos plenamente cuando se sienten respaldados por sus compañeros. Si tenemos en cuenta que durante la adolescencia la relación con los compañeros se vuelve más importante (Goodenow, 1993; Brown y Larson, 2009; Smetana, 2011), el ABP puede ser una buena forma de canalizar la motivación de los alumnos durante la etapa secundaria.

La relación entre alumnos es un elemento esencial en el desarrollo de la motivación académica. De entre las diversas teorías motivacionales existentes, la teoría de la autodeterminación (TAD; Deci y Ryan, 1985, 2000; Ryan y Deci, 2000; Vansteenkiste, Niemiec, y Soenens, 2010; Deci y Ryan, 2014) se caracteriza por otorgar un papel determinante a las relaciones entre alumnos en la construcción de la motivación.

Esta teoría contempla la motivación como un continuo que va desde la ausencia de motivación (amotivación) hasta la motivación intrínseca. El proceso que conduce de una a otra se llama internalización y es promovido mediante el desarrollo de tres necesidades psicológicas básicas: autonomía, competencia y relación con los demás (Deci y Ryan, 1985; Niemiec y Ryan, 2009).

Según la TAD, la autonomía se refiere a la necesidad de desarrollar actividades de forma autorregulada y no heterónoma (Deci y Ryan, 2000). La competencia representa la necesidad de sentirse efectivo frente a acciones concretas, superando los desafíos que aparecen en la vida diaria (LaGuardia y Ryan, 2002). Mientras que la relación con los demás es el establecimiento de relaciones positivas con las personas que influyen en un contexto determinado (Deci y Ryan, 2014). Estos tres elementos se nutren entre sí y, para conseguir una motivación autorregulada, es necesario que se desarrollen de forma equilibrada.

Como señalan Williams, Saizow y Ryan (1999), la TAD permite entender mejor la motivación durante un trabajo cooperativo (como es el caso del ABP), ya que los elementos básicos de este -elección, competencia y colaboración- se pueden entender en términos de autonomía, competencia y relación con los demás.

Aunque las tres necesidades son igualmente importantes, el presente texto se centra en la relación con los demás. En términos motivacionales, la necesidad de relación con los demás se fomenta mediante el grado percibido de conexión con los actores que intervienen en el proceso educativo y mediante el sentimiento de aceptación al grupo (Deci y Ryan, 2000). 
Así, la aceptación entre pares y el apoyo del profesor fomentan la motivación intrínseca en el ámbito académico (Cox, Duncheon y McDavid, 2009).

\section{PROYECTO Y METODOLOGÍA DE INVESTIGACIÓN}

El proyecto objeto de estudio se desarrolló durante un curso escolar en dos grupos de $4^{\circ}$ año de educación secundaria obligatoria (ESO). Este ABP consistió en la adaptación de la obra Seda, de Alessandro Baricco, para teatro con música, danza y video-proyección. A lo largo del proyecto se llevó a cabo una investigación acción que tenía por objetivo mejorar el fomento de las tres necesidades psicológicas básicas. Así, cuando se identificaba un problema en el aula, se realizaba un ciclo de investigación acción en el que se interpretaba el problema en término de necesidades psicológicas básicas para, posteriormente, plantear una solución.

En el primer grupo, la clase de música $(n=12)$, se trabajó en un entorno de interpretación musical clásica en el que todos los alumnos tenían un papel similar y en el que existía una capacidad de decisión muy limitada, ya que el profesor ejercía de director del conjunto instrumental. En el segundo grupo, la clase de artes escénicas $(n=21)$, los alumnos trabajaron en un entorno de mayor autonomía y fueron divididos según la función que desarrollaban en dos subgrupos (actores y bailarines); a principio de curso, estos dos subgrupos ensayaron en aulas separadas y el profesor trabajó presencialmente con cada uno de ellos en días alternos, mientras el otro grupo trabajaba solo.

El objetivo del presente texto es analizar la motivación y la relación con los demás en el contexto de este ABP, teniendo como referencia la TAD. Así, las preguntas principales de investigación son: ¿qué características del proyecto han motivado más a los alumnos?, ¿cómo han valorado la relación con los demás?, ¿qué grado de relación ha existido entre la relación con los demás y las otras dos necesidades psicológicas básicas?

Para responder a las preguntas de investigación se realizaron entrevistas semiestructuradas a los alumnos al acabar el proyecto. Las preguntas se organizaron en torno a las ideas básicas de la TAD y persiguieron conocer los elementos motivacionales que se habían generado durante el desarrollo de este.

Las respuestas han sido sometidas a un análisis de contenido utilizando un enfoque deductivo (Elo y Kyngas, 2008) mediante el programa de análisis NVIVO 12. Como señala Bardin (2001), antes de comenzar el análisis se debe construir una matriz principal de categorización, en nuestro caso hemos tratado de diferenciar entre elementos positivos y negativos en el fomento de cada una de las tres las necesidades básicas durante el desarrollo del proyecto (lo que daría una matriz de $3 \times 2$ ).

\section{RESULTADOS}

La codificación de los temas se realizó teniendo en cuenta las tres necesidades psicológicas básicas. Aunque las preguntas de las entrevistas semiestructuradas ya se habían organizado siguiendo estos tres elementos, las ideas identificadas aparecieron, en muchas ocasiones, de forma transversal. En la Tabla 1 de frecuencias podemos observar una gran diversidad de temas. Es importante señalar que las unidades presentes en el nodo "trabajo sin profesor" 
surgieron de las preguntas realizadas a los alumnos de artes escénicas y hacen referencia al hecho concreto de haber trabajado solos en el aula durante algunas sesiones.

Tabla 1. Frecuencias conjuntas para artes escénicas y música

\begin{tabular}{|c|c|c|c|c|c|c|c|}
\hline \multicolumn{2}{|l|}{ Autonomía } & \multicolumn{2}{|l|}{ Competencia } & \multicolumn{2}{|c|}{ Relación con los demás } & \multicolumn{2}{|c|}{ Trabajo sin profesor } \\
\hline \multicolumn{2}{|l|}{ Positivo } & \multicolumn{2}{|l|}{ Positivo } & \multicolumn{2}{|l|}{ Positivo } & \multicolumn{2}{|l|}{ Negativo } \\
\hline \begin{tabular}{|l|}
$\begin{array}{l}\text { Compromiso } \\
\text { creciente }\end{array}$ \\
\end{tabular} & 36 & $\begin{array}{l}\text { Buen resultado } \\
\text { final }\end{array}$ & 29 & Actuar en público & 9 & $\begin{array}{l}\text { Deficiente trabajo } \\
\text { en equipo }\end{array}$ & 4 \\
\hline \begin{tabular}{|l|} 
Elección \\
percibida positiva
\end{tabular} & 46 & Creatividad & 3 & $\begin{array}{l}\text { Ausencia de } \\
\text { conflictividad }\end{array}$ & 9 & Escasa disciplina & 14 \\
\hline $\begin{array}{l}\text { Valoración } \\
\text { positiva de la } \\
\text { autonomía }\end{array}$ & 12 & $\begin{array}{l}\text { Desarrollo } \\
\text { vocación artística }\end{array}$ & 9 & $\begin{array}{l}\text { Buena relación con } \\
\text { el profesor }\end{array}$ & 4 & Trabajo irregular & 20 \\
\hline \multicolumn{2}{|l|}{ Negativo } & $\begin{array}{l}\text { Mejora dotes } \\
\text { interpretativas }\end{array}$ & 23 & $\begin{array}{l}\text { Relación con los } \\
\text { compañeros }\end{array}$ & 75 & \multicolumn{2}{|l|}{ Positivo } \\
\hline $\begin{array}{l}\text { Autonomía } \\
\text { defecto del } \\
\text { profesor }\end{array}$ & 3 & $\begin{array}{l}\text { Proyecto como } \\
\text { vía de escape }\end{array}$ & 6 & Romper muros aula & 5 & $\begin{array}{l}\text { Ambiente } \\
\text { distendido }\end{array}$ & 1 \\
\hline \multirow[t]{9}{*}{\begin{tabular}{|l|}
$\begin{array}{l}\text { No poder elegir la } \\
\text { obra }\end{array}$ \\
\end{tabular}} & 2 & Práctica artística & 72 & $\begin{array}{l}\text { Trabajo } \\
\text { cooperativo }\end{array}$ & 29 & $\begin{array}{l}\text { Experimentar por } \\
\text { sí mismos }\end{array}$ & 14 \\
\hline & & $\begin{array}{l}\text { Sensación de } \\
\text { competencia }\end{array}$ & 28 & \multicolumn{2}{|l|}{ Negativo } & & \\
\hline & & $\begin{array}{l}\text { Superación miedo } \\
\text { escénico }\end{array}$ & 23 & $\begin{array}{l}\text { Clase muy } \\
\text { numerosa }\end{array}$ & 2 & & \\
\hline & & $\begin{array}{l}\text { Teoría percibida } \\
\text { positivamente }\end{array}$ & 12 & $\begin{array}{l}\text { Conductas } \\
\text { disruptivas leves }\end{array}$ & 19 & & \\
\hline & & $\begin{array}{l}\text { Valoración } \\
\text { positiva del } \\
\text { esfuerzo }\end{array}$ & 16 & $\begin{array}{l}\text { Conflictos leves } \\
\text { compañeros }\end{array}$ & 8 & & \\
\hline & & \multicolumn{2}{|l|}{ Negativo } & Divismo & 4 & & \\
\hline & & $\begin{array}{l}\text { Desentusiasmo } \\
\text { creciente }\end{array}$ & 1 & $\begin{array}{l}\text { Necesidad de más } \\
\text { autoridad docente }\end{array}$ & 4 & & \\
\hline & & Ensayo repetitivo & 15 & $\begin{array}{l}\text { Poca implicación } \\
\text { de pares }\end{array}$ & 14 & & \\
\hline & & $\begin{array}{l}\text { Rechazo de } \\
\text { contenidos } \\
\text { teóricos }\end{array}$ & 19 & $\begin{array}{l}\text { Respuesta negativa } \\
\text { del público }\end{array}$ & 6 & & \\
\hline
\end{tabular}

Las frecuencias de los temas detectados durante el análisis de las entrevistas (Figura 1) muestran un sesgo positivo en las tres necesidades psicológicas básicas. En las dos asignaturas que formaron parte del proyecto (música y artes escénicas), los alumnos destacaron la competencia experimentada por encima de las demás necesidades psicológicas, desarrollando una visión ampliamente positiva sobre la misma. 
Figura 1. Frecuencia de temas relacionados con las tres necesidades psicológicas básicas para las asignaturas de música y de artes escénicas

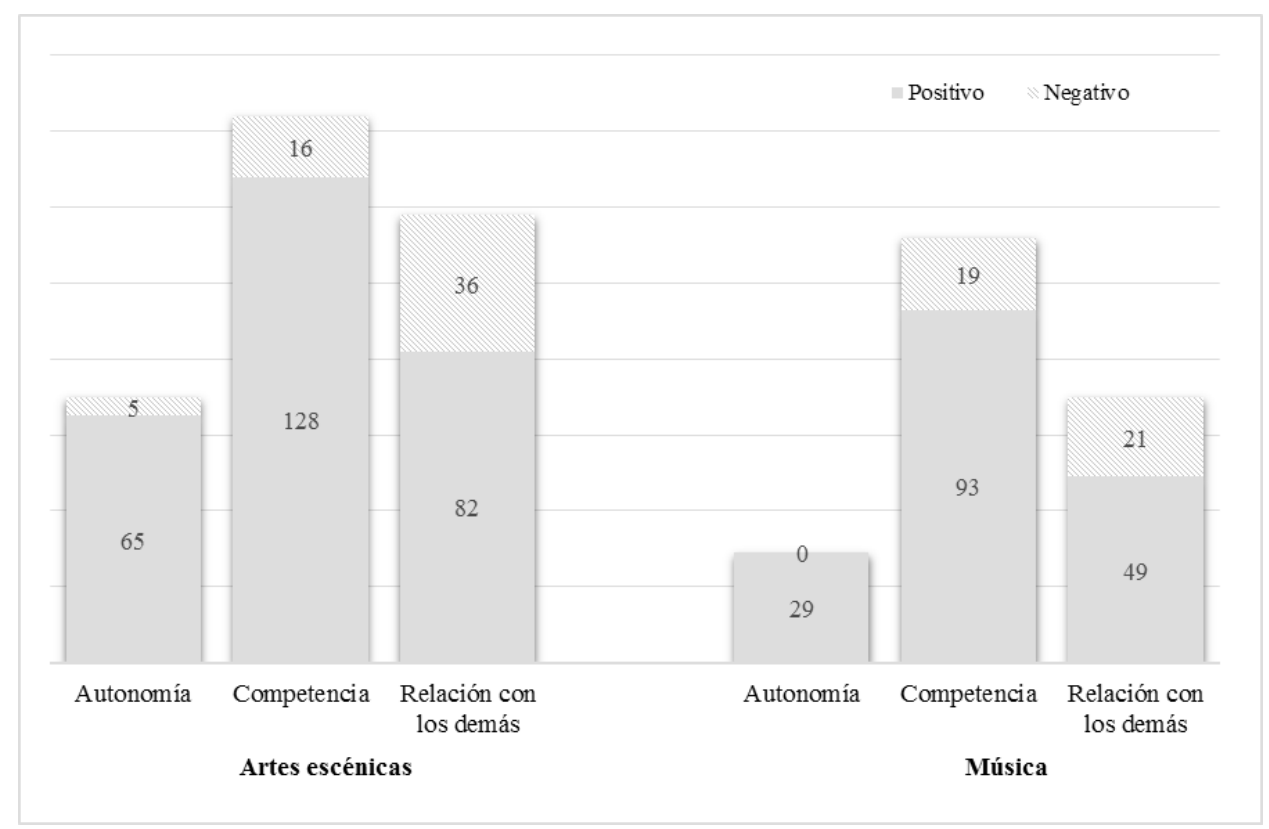

Fuente. Elaboración propia.

En la clase de artes escénicas -donde se concedió mayor capacidad de decisión a los alumnos- tanto la necesidad de autonomía como la de relación con los demás presentaron un sesgo positivo. Los resultados obtenidos para la asignatura de música -cuya metodología se basó en la interpretación musical clásica y ofreció una autonomía muy limitada a los alumnos- no presentaron registros negativos en la necesidad psicológica de autonomía, la que mostró una frecuencia general baja en comparación con el resto de los nodos. La relación con los demás en la clase de música también fue interpretada de forma positiva.

Si enumeramos los temas de mayor a menor frecuencia, podemos observar diferencias entre las asignaturas de artes escénicas y de música. En los gráficos correspondientes (Figuras 2 y 3 ) encontramos los temas valorados de forma positiva a la derecha del eje del gráfico, mientras que los temas valorados de forma negativa se representan con una frecuencia negativa a la izquierda de este.

El tema mejor valorado por los alumnos de artes escénicas (Figura 2) fue la buena relación con los compañeros (incluido en el nodo de relación con los demás), seguido por la práctica artística (incluido en la necesidad psicológica de competencia) y por el compromiso creciente a lo largo del proyecto (relacionado con la necesidad de autonomía). Es decir, los elementos más motivadores para los alumnos de artes escénicas fueron el desarrollo de la relación con sus compañeros, el hecho de realizar un proyecto artístico y el creciente compromiso experimentado conforme se acercaba la fecha del estreno. 
Figura 2. Temas destacados por los alumnos de Artes escénicas

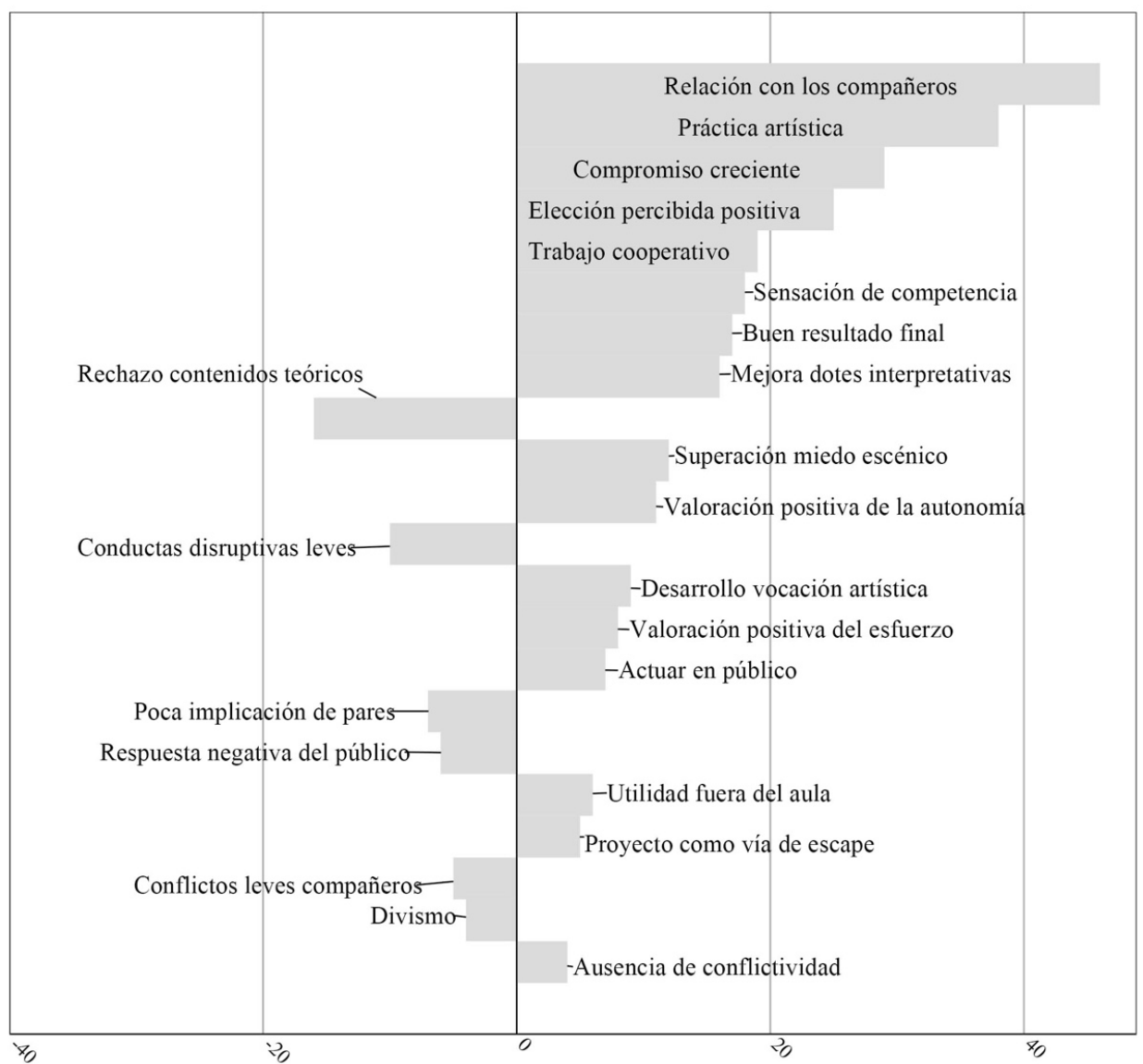

Fuente. Elaboración propia.

Otros temas positivos que obtuvieron una frecuencia alta en las entrevistas de los alumnos de artes escénicas fueron la capacidad de elección percibida, el trabajo cooperativo, la sensación de competencia experimentada, el buen resultado final y la mejora de las dotes interpretativas.

De entre los elementos que socavaron la motivación de los alumnos de artes escénicas destacan el rechazo a los contenidos teóricos que se impartieron a principio de curso, las conductas disruptivas leves y la poca implicación de algunos de algunos compañeros. Además, se subraya la respuesta negativa que tuvo parte el público -compuesto por estudiantes de otros centros- en la segunda representación de la obra, así como los conflictos leves entre compañeros y el divismo manifestado por ciertas personas. No obstante, es importante destacar la menor frecuencia de los elementos negativos respecto de los positivos. 
Figura 3. Temas destacados por los alumnos de música

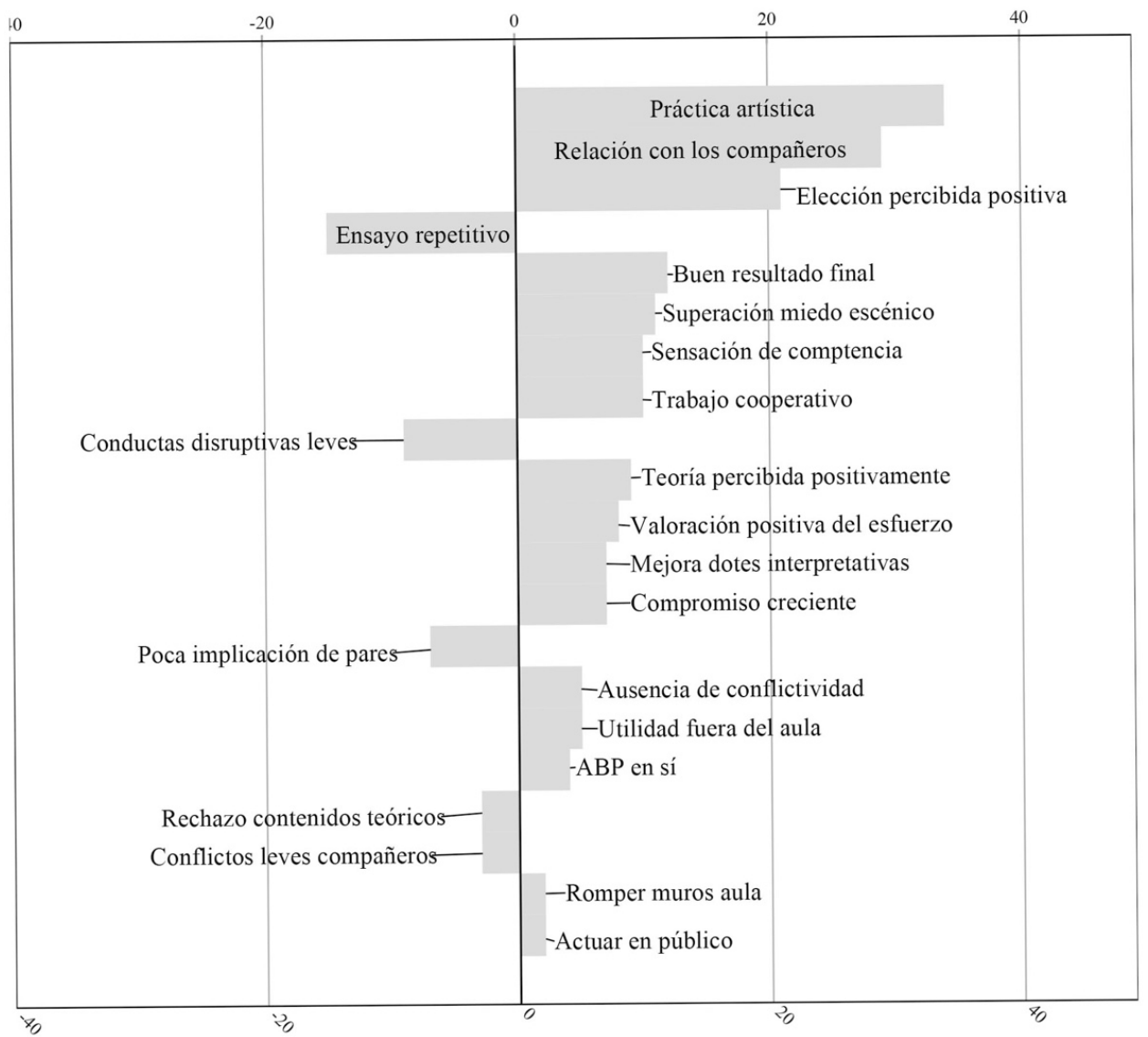

Fuente. Elaboración propia.

En cuanto a los temas destacados por los alumnos de música (Figura 3) la práctica artística ha supuesto el elemento más motivador del proyecto, seguido por la relación con los compañeros. En tercer lugar y a pesar de haber otorgado una capacidad de decisión limitada al grupo, los alumnos destacaron la elección percibida de forma positiva. Otros temas positivos señalados fueron el buen resultado final del proyecto, la superación del miedo escénico, la sensación de competencia y el trabajo cooperativo.

Al contrario que en las frecuencias de los temas destacados por los alumnos de artes escénicas, en las que aparece el primer tema negativo en noveno lugar, el primer elemento negativo destacado por los alumnos de música aparece en cuarta posición: el tema "ensayo repetitivo" hace referencia a la percepción de una dinámica monótona en la interpretación musical. Otros elementos negativos fueron las conductas disruptivas leves, la poca implicación de algunos compañeros, el rechazo a los contenidos teóricos o los conflictos leves. 
Si nos centramos en cómo interpretaron los alumnos la relación con los demás, las respuestas de las entrevistas semiestructuradas muestran un sesgo positivo en cuanto a esta necesidad psicológica básica (Figura 1). Dentro de este nodo, dos de los seis temas más citados destacan claramente por su frecuencia: relación con los compañeros (percibida de forma positiva) y trabajo cooperativo. Más allá del aspecto cuantitativo de las respuestas, las palabras de los alumnos explican las razones por las que ciertos temas tienen una mayor frecuencia. Los fragmentos de las entrevistas que se describen a continuación están anonimizados, es decir, cada uno de los entrevistados se identifica mediante un pseudónimo.

La relación con los compañeros fue mejorando progresivamente conforme avanzaba el proyecto, lo que llevó a una buena percepción de esta a final del curso. Para Aitana, de artes escénicas, la relación entre pares tuvo un balance positivo, a pesar de que al principio no tuviera muchas expectativas al respecto: "pensaba que iba a ser mala y ha sido muy buena. Porque, bueno, yo es que no conocía a nadie" (aita_escenicas_03:25). La asignatura de música, a pesar de seguir una dinámica más rígida basada en la interpretación musical clásica, también permitió un buen desarrollo de la relación con los compañeros. Melisa, al ser preguntada por el aspecto más positivo del proyecto, señala: "una cosa buena, pues que nos hemos conocido más entre todos, nos hemos vuelto más amigos, hemos aprendido cosas nuevas" (mel_musica_04:13).

Además de existir una evolución positiva en la relación entre los compañeros, los alumnos también manifestaron haber desarrollado un "compromiso creciente" a lo largo del proyecto (ver Autonomía-Positiva en Figura 1). En este sentido, Iván, de la asignatura de música, destaca: "coges más confianza con los compañeros. Entonces, eso, quieras o no, ayuda [...] a trabajar mejor" (ivan_musica_00:24). Es decir, el hecho de profundizar en la relación con los pares los llevó a un mejor trabajo grupal.

No obstante, para algunos alumnos existió un obstáculo a la hora de mejorar la relación, a saber, las ansias de protagonismo de algunas personas. Etiquetado bajo el epígrafe "divismo", su frecuencia es muy baja en comparación con el resto de los temas. Así, ciertos alumnos manifestaron haber percibido esta actitud -tan común en el medio artístico- en algunos compañeros. Ángela, por ejemplo, afirma: "hay mucha competitividad, entonces, eso no me hacía disfrutar mucho, porque quieras o no, siempre hay gente que quiere ser la protagonista. A la hora de los bailes hay mucho comportamiento de equipo, pero todos quieren su momento de fama" (ange_escenicas_06:12).

Ahora bien, esta reflexión es minoritaria entre el grupo y la mayoría de los alumnos destaca la buena relación con los compañeros. Para José, el hecho de no conocer a la gente hace que exista cierta rivalidad, pero el contacto y la mejora de la relación lleva a que la sensación de divismo hacia otras personas se disipe:

"al principio, si no hay confianza, claro que te va a costar un montón [trabajar], sobre todo decidir lo que tienes que hacer. Va a haber como una falta de confianza y tú vas a querer ser primero o va a haber como luchas o competencias por ver quién va a estar en primera fila o en última, quién va a ser el personaje o no. Pero al final tú vas conociendo a la gente y te va cayendo bien y dices bueno, este lo hará mejor que yo" (jose_escenicas_03:35).

Es importante señalar que todas las referencias al divismo se produjeron en el grupo de artes escénicas. Este hecho puede deberse a que en la clase de música todos los alumnos 
tenían un papel similar, a excepción de dos canciones en las que dos chicas tomaron un mayor protagonismo.

En términos generales, tanto para los alumnos de música, como para los de artes escénicas, la buena relación entre pares -junto a la práctica artística- fue uno de los aspectos más motivadores del proyecto. Como señala Irene, "[esta buena relación] ha sido casi lo mejor de la experiencia de la obra" (irene_escenicas_00:51). Las palabras de Alicia también enlazan este aspecto con el desarrollo de la motivación intrínseca: "nos hemos unido mucho y yo creo que todos nos hemos quedado con ganas de hacer más proyectos" (ali_escenicas_06:11).

Otro de los puntos destacados de forma positiva fue el carácter colaborativo del proyecto. Iván, de la asignatura de música, asegura: "yo creo que hemos aprendido a trabajar en grupo, porque, quieras o no, no siempre se trabaja en grupo" (ivan_musica_03:03). En el buen desarrollo del trabajo cooperativo influyó el respeto que se profesaban los alumnos entre sí. Alicia, de artes escénicas, afirma: "todos hemos tenido nuestro momento, nuestra opinión y, por suerte, estábamos en un grupo en que todas las opiniones se escuchaban y se tenían bastante en cuenta" (ali_escenicas_04:27). Esta opinión no solo se desarrolló en la clase de artes escénicas, sino también en la de música, donde alumnos como Melisa destacan la necesidad de escuchar y de ser escuchado al realizar un trabajo grupal: "[en el trabajo en grupo] conoces a más gente, eres capaz de escuchar la opinión de los demás y no solo la tuya porque puede que tú estés equivocado en algunas cosas" (mel_musica_06:18).

Por otra parte, la idea del trabajo en equipo se relaciona con la autonomía otorgada por el profesor. La capacidad de elección de los alumnos fue mayor en la clase de artes escénicas, por lo que las decisiones tomadas por ellos mismos tuvieron una mayor repercusión en la dinámica del grupo. Clara, al ser preguntada sobre si le parecía bien que los bailarines crearan algunas coreografías por su cuenta y sin ayuda del profesor, afirmaba: "así estábamos de acuerdo en algo [...] cuando lo marcabas tú, bien, pero cuando lo hacíamos nosotros era como que todos estábamos de acuerdo" (clara_escenicas_03:05). Irene, también defiende esta forma de trabajar: "en las coreografías sí que podíamos opinar y decir aquello que no nos gustaba y que se podía cambiar y eso ha estado muy bien, porque así cada uno ponía su granito" (irene_escenicas_02:30).

A la buena relación con los compañeros y al trabajo colaborativo se suma la ausencia de conflictividad entre los alumnos como tema destacado de forma positiva. Además, se han identificado otros temas de menor frecuencia que han ayudado al desarrollo de la relación con los demás. Estos temas, aunque secundarios, permiten entender la potencialidad del $\mathrm{ABP}$ en el desarrollo de esta necesidad psicológica básica. De mayor a menor importancia, podemos destacar el hecho de haber actuado en público, de haber tenido contacto con personas de fuera del centro (ítem titulado "romper muros del aula") o de haber establecido una buena relación con el profesor.

En cuanto a los temas destacados de forma negativa, las conductas disruptivas leves, la poca implicación de algunos alumnos y, en menor medida, los conflictos leves entre compañeros suponen los principales problemas detectados por los alumnos en cuanto al desarrollo de la necesidad psicológica de relación con los demás.

Las conductas disruptivas leves estuvieron presentes a lo largo de todo el curso en ambas asignaturas $\mathrm{y}$, aunque no subieron de tono (ningún alumno fue amonestado o expulsado durante el transcurso del proyecto), sí que minaban la concentración del grupo. Bryan, de música, al ser preguntado por algún elemento negativo de la dinámica de clase, 
respondía: "cuando alguien tocaba cuando tú decías que se callasen, me irritaba" (bryan_ musica_00:35). En la clase de música, efectivamente, estas conductas disruptivas leves estaban relacionadas con hablar fuera de turno o tocar los instrumentos cuando el profesor estaba ofreciendo una explicación a otros alumnos. En definitiva, el problema se traducía en una pérdida de tiempo de trabajo: "porque en lugar de aprovechar el tiempo como era debido -sentencia Melisa- pues perdíamos más tiempo, en plan, con broncas" (mel_ musica_06/18).

A pesar de esto, para Lluís, que asistía a ambas asignaturas, el ambiente en música era diferente al de artes escénicas: “[en música] estaba más controlado, éramos menos gente, también” (lluis_musica_04:15). En artes escénicas el problema residía en que, mientras unos alumnos bailaban o actuaban, otros debían esperar al momento en que salían a escena. En este sentido Alba afirma: "cuando hacíamos generales [...] hay gente que no bailaba en esa escena, y pues lo único que hacíamos era hablar" (alba_escenicas_03:46). Este comportamiento tenía una incidencia negativa sobre la motivación del grupo. Irene, al hacer balance sobre los aspectos negativos del proyecto señala: “[una cosa] mala, pues, por una parte, en todos los ensayos no me lo he pasado tan bien porque había igual gente que molestaba" (irene_escenicas_03:33).

La dinámica de la clase de artes escénicas incluía que bailarines y actores trabajasen, al principio, por separado, por lo que el profesor pasaba de un aula a otra para verificar el avance de los grupos. Para muchos alumnos, esta dinámica no funcionaba bien: "cuando estábamos en clase que nos dejabas solos -señala María M.- había algunos que sí que eran más responsables, pero otros que pasaban de todo y algunos, pues que entretenían a las personas" (mam_escenicas_02:35). Así, el otorgar una autonomía casi total durante algunas partes del ensayo tenía una repercusión negativa en la relación con los demás.

Además de las conductas disruptivas leves, los alumnos identificaron la falta de implicación de ciertos compañeros como un elemento negativo. Esta actitud se identificó sobre todo a principio de curso, ya que los alumnos manifestaron un "compromiso creciente" a lo largo del proyecto (ver Autonomía-positivo en Figura 1). Para Reychel, de artes escénicas, "a algunos, como que les daba igual hacerlo, no ponían de su parte y al final, que vieron que ya teníamos que presentarla, ya empezaron a trabajar" (reychel_ escecnicas_04:36). Los alumnos del grupo de música también percibieron esta actitud al comenzar el proyecto: "[una cosa mala del proyecto] sería esa gente que no se lo tomó en serio desde el principio - afirma Eugenia S.- que al final la cosa ha salido bien, pero, era un poco desesperante, sobre todo al principio" (euges_musica_02:45).

Otro tema destacado de forma negativa en ambos grupos -aunque con una frecuencia muy baja- fue la aparición de conflictos leves entre compañeros. No obstante, no se identificaron problemas mayores y cada uno de estos conflictos se resolvió sin intervención del profesor. Alicia, de artes escénicas, al ser preguntada sobre si hubo de conflictos importantes entre sus compañeros afirma: "pues, que yo recuerde no. Fuertes, problemas fuertes yo creo que ninguno. Solo diferencias" (ali_escenicas_01:16).

A pesar de las bajas frecuencias de los temas negativos citados, es importante señalar que la relación con los demás es la necesidad psicológica básica que más ítems negativos produce. En el caso de los alumnos de artes escénicas, el tema negativo más citado es el rechazo a los contenidos teóricos vistos a principio de curso, mientras que los cinco siguientes ítems se relacionan con dicha necesidad psicológica: conductas disruptivas leves, poca implicación de pares, respuesta negativa del público (en una de las representaciones, el 
público, formado por alumnos de otro centro, molestó considerablemente), conflictos leves entre compañeros y divismo. Los alumnos de música, por su parte, destacan lo repetitivo de los ensayos como el aspecto más negativo del proyecto, seguido por las conductas disruptivas leves y la poca implicación de algunos pares.

\section{DISCUSIÓN}

El análisis de las entrevistas semiestructuradas muestra la importancia que tuvo el desarrollo de la necesidad psicológica de relación con los demás sobre la motivación. Esta relación no solo generó resultados positivos, sino también negativos. Por un lado, los alumnos valoraron la buena relación con los demás como uno de los elementos más positivos del proyecto, tanto en la clase de música como en la de artes escénicas; en sentido contrario, entre los temas negativos de mayor frecuencia destacaron el mal comportamiento y la poca implicación de algunos compañeros. Como señala Epstein (1983; cit. en Goodenow, 1993), la adolescencia es la etapa en la que los alumnos son más influenciables por el grupo de pares, por esto, los compañeros pueden tener una influencia tanto positiva como negativa sobre la motivación, el compromiso y el éxito escolares.

El principal problema relacional detectado por los alumnos fue el desarrollo de conductas disruptivas leves. Como muestra el análisis de las entrevistas, este tipo de comportamiento minaba la motivación del grupo. No obstante, su existencia puede ser enmarcada dentro de la problemática general de la educación secundaria. Tanto los profesores como los alumnos de esta etapa educativa señalan que hablar fuera de turno supone el principal elemento disruptivo en la dinámica de clase (Houghton, Wheldall y Merrett, 1988; Beaman, Wheldall y Kemp, 2007; Sun y Shek, 2013).

$\mathrm{Si}$, a priori, puede parecer que el profesor es el más preocupado por la falta de disciplina, nuestros resultados muestran que los alumnos sienten este problema como propio. Este hecho también es destacado por Fernández-Balboa (1991, cit. en Hodges, Cothran y Regualos, 2006), quien afirma que el mal comportamiento de algunos alumnos no solo distrae al profesor sino también a los propios discentes.

Otro tema negativo que hacía referencia a la relación con los demás fue la poca implicación de ciertos compañeros, sobre todo a principio del proyecto, lo cual resulta especialmente importante en un ABP, ya que el trabajo de cada alumno influye sobre el resultado general. Así, los alumnos interpretan la desgana de alguno de sus pares como una amenaza hacia su propio rendimiento. No obstante, este comportamiento también puede enmarcarse dentro de la problemática general de los adolescentes en el aula: Little (2005) demostró, que, cuando los alumnos alcanzan los últimos cursos de educación secundaria (nuestro proyecto se desarrolló en el último curso de ESO), la segunda conducta negativa más citada por los profesores pasa a ser la pereza del discente, solo superada por el hecho de hablar fuera de turno.

A pesar de esta percepción negativa sobre ciertos compañeros, el conjunto del proyecto generó un clima positivo de relación entre pares. Así, como manifestaron los alumnos durante las entrevistas, la relación fue mejorando a lo largo del proyecto. De forma paralela, la implicación del grupo fue ganando fuerza hasta la representación final (ver el tema compromiso creciente), en la que se consiguió un buen producto artístico. Esta correlación entre mejora de la relación, mejora del trabajo y resultado positivo, podría explicarse de 
forma causal según estudios previos en los que se han demostrado que el desarrollo de la relación con los demás fomenta la implicación y el rendimiento académicos (Kindermann, McCollam y Gibson, 1996; Furrer y Skinner, 2003; Mikami, Ruzek, Hafen, Gregory y Allen, 2017).

El hecho de que los alumnos de ambos grupos desarrollaran un compromiso creciente, percibieran un buen resultado final y destacaran la práctica artística como uno de los elementos más importantes del proyecto, indica que el objetivo final de este -el vivenciar la experiencia escénica- fue interiorizado por la mayoría de ellos de manera exitosa. Si, como afirman Wentzel y Wigfield (1998), para que exista un logro académico, los objetivos del alumno deben corresponderse con los objetivos del grupo, podemos afirmar que nuestros alumnos han integrado sus objetivos individuales con aquellos del proyecto. Este proceso es definido por la teoría de la autodeterminación en términos de internalización de la conducta, lo que se asocia a un desarrollo de la motivación (Niemiec y Ryan, 2009).

Así, se ha conseguido un trabajo cooperativo eficiente, fomentado una interdependencia positiva (Johnson, Johnson y Holubec, 1994), en el que cada esfuerzo individual se ha encaminado a la consecución del objetivo colectivo y donde cada miembro ha realizado una contribución única. Según varios autores (Martin y Dawson, 2009; Hardré et al., 2006) el aprendizaje cooperativo -y el ABP en esencia lo es (Liu, Wang, Tan, Koh y Ee, 2009)ayuda a desarrollar la relación con los demás, por lo que los buenos resultados obtenidos en la percepción de relación con los demás de nuestros alumnos podrían explicarse por el buen trabajo cooperativo llevado a cabo.

Por otro lado, también se ha observado una influencia de la relación con los demás sobre la competencia percibida. Uno los temas relacionados con esta necesidad psicológica ha sido la superación del miedo escénico. En este sentido, la buena relación con los demás manifestada por los alumnos puede haber contribuido a la seguridad de estos en el escenario: como señalan Hamm y Faircloth (2005), la buena relación en el contexto del aula de secundaria facilita la toma de riesgos por parte del alumno sin el temor de ser objeto de burlas.

Por último, hay que ahondar en la interacción entre la relación con los demás y la autonomía percibida. Al igual que con las otras dos necesidades psicológicas básicas, la percepción general de autonomía durante el proyecto fue positiva en los dos grupos estudiados. Únicamente durante el tiempo en el que el grupo de artes escénicas trabajó sin profesor, los alumnos tuvieron la sensación de no haber trabajado bien. El no tener al profesor para controlar la dinámica de grupo, conllevó la imposibilidad de implementar un estilo docente de apoyo a la autonomía (Deci, Eghrari, Patrick, y Leone, 1994; Reeve, 2009; 2016), lo cual resulta esencial a la hora de mejorar la sensación de autonomía. El trabajo sin profesor reveló que, sin la creación de un contexto apropiado para trabajar y relacionarse, existieron más conductas disruptivas.

Cuando los alumnos trabajan juntos -con ayuda del profesor- para cooperar en proyectos y compartir sus ideas crean un contexto de apoyo a la autonomía (Furrer, Skinner y Pitzer, 2014). Así, los contextos de apoyo a la autonomía impulsan la relación con los demás (Ryan y Powelson, 1991) y predicen una mayor motivación y un mayor bienestar (Chirkov y Ryan, 2001).

Si hablamos del conjunto del proyecto -a excepción de los momentos en los que se trabajó sin profesor- podemos afirmar que se desarrolló un contexto de apoyo a la autonomía, evitando un contexto de control. Como señalan Vansteenkiste et al. (2010), 
el estilo controlador presenta tres condiciones: primero, el profesor se entromete en las acciones, pensamientos y sentimientos de los alumnos, segundo, adopta su punto de vista de forma única y, tercero, presiona a los discentes para pensar, sentir o actuar de una determinada forma. En el análisis de contenido no se han identificado temas que se asocien a un estilo controlador por parte del profesor, por lo que se verifica que ha primado un estilo de apoyo a la autonomía. En este sentido, los alumnos han desatacado la buena relación con el profesor e, incluso, han manifestado la necesidad de más autoridad por su parte.

En definitiva, el proyecto consiguió desarrollar las tres necesidades psicológicas básicas de forma equilibrada. Como afirman Deci y Ryan (2014), la necesidad de relación con los demás no asegura por sí misma relaciones de calidad y la motivación, a no ser que vaya acompañada del desarrollo de las otras dos necesidades psicológicas básicas, competencia y autonomía.

\section{CONCLUSIONES}

Aunque la relación con los demás fue la necesidad psicológica que más frecuencias negativas presentó, uno de los aspectos más motivadores del proyecto fue la buena relación desarrollada entre alumnos. La dinámica del grupo de artes escénicas -en el que estos interactuaban de una forma más directa- favoreció especialmente esta necesidad. A pesar de que en el grupo de música hubo menor capacidad de elección y primó una dinámica más rígida, existió una percepción muy positiva de la relación entre pares. Los aspectos negativos referentes a la relación con los demás se concretaron en la aparición de conductas disruptivas leves y en la poca implicación de algunos compañeros.

El desarrollo de la necesidad de competencia afectó positivamente a la relación con los demás. Así, los temas negativos identificados a principio de curso se atenuaron conforme el grupo experimentaba una mayor competencia y un mejor trabajo cooperativo. Por su parte, la creación de un contexto de apoyo a la autonomía también fomentó la relación entre compañeros. Únicamente durante el trabajo sin profesor, donde no se pudo ofrecer este contexto, los alumnos desarrollaron un trabajo más irregular y mayores problemas de relación.

En definitiva, el ABP analizado permitió tanto la mejora de la relación con los demás, como el desarrollo de las otras dos necesidades psicológicas básicas, competencia y autonomía. Así, se creó el contexto idóneo para, según la TAD, alcanzar una motivación intrínseca.

\section{REFERENCIAS BIBLIOGRÁFICAS}

Beaman, R., Wheldall, K. \& Kemp, C. (2007). Recent research on troublesome classroom behavior: a review.Australasian JournalofSpecialEducation, 31(1),45-60. DOI: 10.1080/10300110701189014

Bardin, L. (2001). L'analyse de contenu. Paris: Presses Universitaires de France.

Brown, B. B. \& Larson, J. (2009). Peer relationships in adolescence. En R. M. Lerner \& L. Steinberg (Eds.), Handbook of adolescent psychology (pp. 74-103). Nueva York: Wiley.

Cox, A., Duncheon, N., \& McDavid, L. (2009). Peers and teachers as sources of relatedness perceptions, motivation, and affective responses in physical education. Research Quarterly for 
Exercise and Sport, 80(4), 765-773. DOI: 10.1080/02701367.2009.10599618

Chirkov, V. I. \& Ryan, R. M. (2001). Parent and teacher autonomy-support in Russian and US adolescents: Common effects on well-being and academic motivation. Journal of Cross-Cultural Psychology, 32(5), 618-635. https://doi.org/10.1177/0022022101032005006

Deci, E. L., Eghrari, H., Patrick, B. C. \& Leone, D. R. (1994). Facilitating internalization: The self-determination theory perspective. Journal of Personality, 62(1), 119-142. http://dx.doi. org/10.1111/j.1467-6494.1994.tb00797.x

Deci, E. L. \& Ryan, R. M. (1985). Intrinsic motivation and self-determination in human behavior. Nueva York, NY: Plenum.

. (2000). The "What" and "Why" of goal pursuits: human needs and the self-determination of behavior. Psychological Inquiry. 11(4), 227-268. DOI: 10.1207/S15327965PLI1104_01

. (2014). Autonomy and need satisfaction in close relationships: Relationships motivation theory. En N. Weinstein (Ed.), Human motivation and interpersonal relationships: Theory, research, and applications (pp. 53-739). Dordrecht: Springer.

Elo, S. \& Kyngas, H. (2008). The qualitative content analysis process. Journal of Advanced Nursing, 62(1), 107-115. DOI: 10.1111/j.1365-2648.2007.04569.x

Epstein, J. (1983). School environment and student friendships: issues, implications and interventions. En J. Epstein \& N. Karweit (Eds.), Friends in school (pp. 235-253). Nueva York: Academic Press.

Fernández-Balboa, J. M. (1991). Beliefs, interactive thoughts, and actions of physical education students' teachers regarding pupil misbehaviors. Journal of Teaching in Physical Education, 11(1), 59-78.

Furrer, C. \& Skinner, E. (2003). Sense of relatedness as a factor in children's academic engagement and performance. Journal of Educational Psychology, 95(1), 148-162. DOI: http://dx.doi. org/10.1037/0022-0663.95.1.148

Furrer, C. J., Skinner, E. A., \& Pitzer, J. R. (2014). The influence of teacher and peer relationships on students' classroom engagement and everyday motivational resilience. In D. Shernoff, \& J. Bempechat (Eds.), National Society for the Study of Education yearbook: Engaging youth in schools: Evidence-based models to guide future innovations (pp. 101-123). New York, NY: Teachers College Record.

Goodenow, C. (1993). Classroom belonging among early adolescent students. Journal of Early Adolescence, 13(1), 21-43. DOI: https://doi.org/10.1177/0272431693013001002

Hamm, J. V. \& Faircloth, B. S. (2005). Peer context of mathematics classroom belonging in early adolescence. The Journal of Early Adolescence, 25(3), 345-366. DOI: https://doi. org/10.1177/0272431605276932

Hardré, P. L., Chen, Ch. H., Huang, S. H., Chiang, Ch. T., Jen, F. L. \& Warden, L. (2006). Factors affecting high school students' academic motivation in Taiwan. Asia Pacific Journal of Education, 26(2), 189-207. DOI: 10.1080/02188790600937326

Helle, L., Tynjälä, P. \& Olkinuora, E. (2006). Project-based learning in post-secondary education - theory, practice and rubber sling shots. Higher Education, 51(2), 287-314. DOI: https://doi. org/10.1007/s10734-004-6386-5

Hodges, P., Cothran, D. \& Regualos, R. (2006). Teachers' reports of student misbehavior in physical education. Research Quarterly for Exercise and Sport, 77(1), 32-40. DOI: 10.1080/02701367.2006.10599329

Houghton, S., Wheldall, K. \& Merrett, F. (1988). Classroom behavior problems which secondary school teachers say they find most troublesome. British Educational Research Journal, 14(3), 297-312. DOI: $10.1080 / 0141192880140306$

Johnson, D. W., Johnson, R. T. \& Holubec, E. J. (1994). The new circles of learning: Cooperation in the classroom and school. Alexandria, VA: Association for Supervision and Curriculum Development. 
Kindermann, T. A., McCollam, T. L. \& Gibson Jr., E. (1996). Peer networks and students' classroom engagement during childhood and adolescence. En K. Wentzel \& J. Juvonen (Eds.) Social Motivation: Understanding children's school adjustment (pp. 279-312). Cambridge: Cambridge University Press.

Kokotsaki, D., Menzies, V. \& Wiggins, A. (2016). Project-based learning: A review of the literature. Improving Schools, 19(3), 267-277. DOI: https://doi.org/10.1177/1365480216659733

LaGuardia, J. G. \& Ryan, R. M. (2002). What adolescents need: A self-determination theory perspective on development within families, school and society. En F. Pajares \& T. Urdan (Eds.), Academic motivation of adolescents (vol. 2, pp. 193-220). Greenwich CT: IAP.

Little, E. (2005). Secondary school teachers' perceptions of students' problem behaviors. Educational Psychology, 25(4), 369-377. DOI: 10.1080/01443410500041516

Liu, W. C., Wang, C. K. J., Tan, O. S., Koh, C. \& Ee, J. (2009). A self-determination approach to understanding students' motivation in project work. Learning and Individual Differences, 19(1), 139 - 145. DOI: https://doi.org/10.1016/j.lindif.2008.07.002

Mac-Iver, D. J., Young, E. M. \& Washburn, B. (2002). Instructional practices and motivation during middle school (with special attention to science). En Wigfield, A. \& Eccles, J. (eds.), Development of achievement motivation (pp. 333-351). San Diego, CA: Academic Press.

Martin, A. J. \& Dawson, M. (2009). Interpersonal relationships, motivation, engagement, and achievement: yields for theory, current issues, and educational practice. Review of Educational Research, 79(1), 327-365. DOI: doi:10.3102/0034654308325583.

Mergendoller, J., Markham, T., Ravitz, J. \& Larmer, J. (2006). Pervasive management of projectbased learning. En C. Evertson \& S. Weinstein (Eds.), Handbook of classroom management: Research, practice, and contemporary issues (pp. 583-615). Mahwah: Lawrence Erlbaum.

Mikami, A. Y., Ruzek, E. A., Hafen, C. A., Gregory, A. \& Allen, J. P. (2017). Perceptions of relatedness with classroom peers promote adolescents' behavioral engagement and achievement in secondary school. Journal of Youth and Adolescence, 46(11), 2341-2354. DOI: 10.1007/ s10964-017-0724-2

Niemiec, Ch. N. \& Ryan, R. M. (2009). Autonomy, competence, and relatedness in the classroom: applying self-determination theory to educational practice. Theory and Research in Education. 7(2), 133-144. DOI:10.1177/1477878509104318.

Qin, Z., Johnson, D. W. \& Johnson, R. T. (1995). Cooperative versus competitive efforts and problem solving. Review of Educational Research, 65(2), 129-143. DOI: 10.3102/00346543065002129.

Reeve, J. (2009). Why teachers adopt a controlling motivating style toward students and how they can become more autonomy supportive. Educational Psychologist, 44(3), 159-175. DOI: $10.1080 / 00461520903028990$

. (2016). Autonomy-supportive teaching: What it is, how to do it. En W. Liu, J. Wang \& R. M. Ryan (eds.) Building Autonomous Learners (pp. 129-152). Singapore: Springer.

Ryan, R. M. \& Deci, E. L. (2000). Self-determination Theory and the facilitation of intrinsic motivation, social development, and well-being. American Psychologist, 55(1), 68-78. DOI: http://dx.doi.org/10.1037/0003-066X.55.1.68

Ryan, R. M. \& Powelson, C. L. (1991). Autonomy and relatedness as fundamental to motivation and education. The Journal of Experimental Education, 60(1), 49-66. DOI: http://dx.doi.org/10.108 0/00220973.1991.10806579

Ryan, R. M., Stiller, J. D. \& Lynch, J. H. (1994). Representations of relationships to teachers, parents, and friends as predictors of academic motivation and self-esteem. The Journal of Early Adolescence, 14(2), 226-249. DOI:10.1177/027243169401400207.

Smetana, J. (2011). Adolescent relationships and development within and between cultures. En J. Smetana (Ed.), Adolescents, families, and social development: How teens construct their worlds (pp. 120-138). Oxford: Wiley.

Stefanou C, Stolk J. D., Prince M., Chen J. C. \& Lord, S. M. (2013). Self-regulation and autonomy in 
problem-and project-based learning environments. Active Learning in Higher Education, 14(2), 109-22. DOI:10.1177/1469787413481132.

Sun, R. C. F. \& Shek, D. T. L. (2013). Classroom misbehavior in the eyes of students: A qualitative study. International Journal of Child Health and Human Development, 6(1), 113-123. DOI: http://dx.doi.org/10.1100/2012/398482

Thomas, J. W. (2000). A review of research on project-based learning. San Rafael, CA: Autodesk Foundation.

Vansteenkiste, M., Niemiec, C. P. \& Soenens, B. (2010). Self-determination theory: an historical overview, emerging trends, and future directions. En S. Karabenick \& T. C. Urdan (Eds.). Advances in motivation and achievement, 16A: decade ahead (pp.105-166). Bradford: Emerald Group Publishing Limited.

Wentzel, K. R. \& Wigfield, A. (1998). Academic and social motivational influences on students' academic performance. Educational Psychology Review, 10(2), 155-175. DOI: https://doi. org/10.1023/A:1022137619834

Williams, G. C., Saizow, R. B. \& Ryan, R. M. (1999). The importance of self-determination theory for medical education. Academic Medicine, 74(9), 972-979. DOI: 10.1097/00001888-19990900000010 